\title{
Food Literacy and Its Associated Factors in Non-Dialysis Patients with Chronic Kidney Disease in China: A Cross-Sectional Study
}

\author{
Di-Fei Duan $\mathbb{1}^{1,2, *}$, Min Liu ${ }^{1,2, *}$, Yi Chen ${ }^{1,2}$, Yue-Yang Huang ${ }^{1,2}$, Yun-Ying Shi ${ }^{1}$ \\ 'Department of Nephrology, West China Hospital of Sichuan University, Chengdu, People's Republic of China; ${ }^{2}$ West China School of Nursing, \\ Sichuan University, Chengdu, People's Republic of China \\ *These authors contributed equally to this work
}

Correspondence: Yun-Ying Shi, Department of Nephrology, West China Hospital of Sichuan University, No. 37 Guoxuexiang Wuhou District, Chengdu, Sichuan, People’s Republic of China, Email yyshi0I30@I63.com

\begin{abstract}
Purpose: Nephrology professionals' understanding of food literacy (FL) and influencing factors is significant for nutrition management, which is key to controlling disease progress among non-dialysis patients with chronic kidney disease (CKD). However, few studies have explored FL in CKD patients. Hence, this study aimed to investigate the level of FL of non-dialysis patients with CKD and to analyze influencing factors in China.

Patients and Methods: A total of 203 CKD patients without dialysis were recruited from August to December 2020 to participate in a cross-sectional study. Food literacy was assessed by a modified short food literacy questionnaire for adults. Related influencing factors were measured by the motivation for dietary self-control scale and the satisfaction with dietary behavior scale. Data were also collected regarding patients' health information-seeking behavior (four items), satisfaction and compliance with dietary advice from healthcare professionals (two items), and demographics and clinical diagnoses.

Results: The mean age of patients was 44.5 years (range 18 to 75 ), and $42.5 \%$ were male. Most (50.2\%) were in stages $1-2$ of CKD. The mean FL score of these patients was $38.75 \pm 0.38$. The multivariable linear regression analysis shows that secondary educational level $(\beta=0.221, \mathrm{p}=0.004)$, motivation for controlling diet $(\beta=0.198, \mathrm{p}=0.003)$, satisfaction with dietary behaviors $(\beta=0.319, \mathrm{p}<0.001)$, and health information-seeking behavior $(\beta=0.146, \mathrm{p}=0.019)$ were significant influencing factors.

Conclusion: Food literacy of Chinese CKD patients without dialysis should be improved. Patients with higher education levels exhibit more active information-seeking behaviors, have greater satisfaction with dietary behaviors, and their motivation for dietary self-control is more likely to be associated with better FL. Healthcare workers should be aware of factors influencing FL and attempt to integrate assessment of FL into routine food-related education for CKD patients.
\end{abstract}

Keywords: motivation, satisfaction, information-seeking behavior, health literacy, nutrition

\section{Introduction}

Chronic kidney disease (CKD) is characterized by a progressive and irreversible loss of renal function. ${ }^{1}$ CKD has been a global public health problem for many decades. ${ }^{2}$ The global all-age mortality rate caused by CKD increased $41.5 \%$ [95\% Uncertainty interval: 35.2 to 46.5] between 1990 and 2017. ${ }^{3}$ Due to its irreversible nature, CKD may progress into end-stage kidney disease (ESKD), and patients may eventually need dialysis therapy or renal transplantation to extend their lifespan. Therefore, effective management is critical to delaying the progression of the illness and the initiation of dialysis treatment. ${ }^{4}$ In the CKD population, nutritional status is often problematic requiring dietary adjustments. ${ }^{5}$ Diet is essential to almost every aspect of care, especially for patients in early stages of $\mathrm{CKD}^{6}{ }^{6}$ As a result, the dietary regimens for CKD are almost the most restrictive among those for any chronic disease. ${ }^{7}$ 
Kidney/Disease Outcome Quality Initiatives suggest that routine management of CKD patients should incorporate dietary guidance from specialist dieticians or multidisciplinary nephrology teams. Patients should receive individualized and comprehensive diet plans. ${ }^{8}$ In addition to specific dietary regimens, patients' healthy dietary patterns also play a significant role in mitigating CKD progression, ${ }^{9}$ but dietary recommendations often do not consider the complex system that influences patients' readiness to change their food choices. As a result, the low adherence in dietary intervention studies is not surprising, given that food choice decisions are usually context-dependent, multideterminant, complex, and dynamic phenomena influenced by social, psychological, and environmental factors. ${ }^{10}$ Nutritional management is thus particularly challenging for both educators (nephrology professionals) and patients. ${ }^{11}$

It is well-known that health literacy is significant for chronic disease and its treatment. ${ }^{12,13}$ For CKD patients, health literacy is associated with transplant waitlist mortality and unhealthy lifestyle behaviors. ${ }^{14,15}$. Recently, the term food literacy (FL), a special form of health literacy, has started to attract the attention of healthcare professionals. ${ }^{16} \mathrm{FL}$ conceptualizes the knowledge, skills, and behaviors needed to achieve a high-quality diet and covers planning and management, selection, preparation, and cooking and eating. ${ }^{16}$ In other words, FL helps an individuals to feed themselves in a way that promotes good nutritional status. Studies have indicated that FL can be modified to improve health, because people with higher FL can make better food choices than those with limited FL. ${ }^{17}$ For example, among patients with heart failure, those who have higher levels of FL have lower sodium consumption, improved control of their disease, and higher quality of life. ${ }^{18}$ The qualitative study by Karin et al reported FL as a facilitator of adherence to dietary guidance and demonstrated a link to increased fruit and vegetable consumption in renal transplant recipients. ${ }^{19}$ Additionally, lower FL is associated with food insecurity that increases the likelihood of progression from CKD to ESKD. ${ }^{20,21}$ Therefore, nephrology professionals' understanding of FL and its influential role among CKD patients appears to be significant for nutrition management.

Psychological factors, health and learning contexts are all crucial aspects influencing FL. ${ }^{22}$ Some researchers have highlighted the importance of associating psychological factors (such as motivation and empowerment) with practical skills related to food. ${ }^{23,24}$ Maartje et al have also recognized the impact of FL on health and well-being in physical and psychological terms. ${ }^{25}$ Regarding learning context, health information-seeking behavior (HISB) and professional support are the main factors influencing FL. Considering well-known associations between health literacy and HISB and communication with healthcare professionals, ${ }^{26,27}$ it is reasonable to hypothesize that HISB will impact FL.

FL is closely related to patient care and should be efficiently explored in order to modify the dietary interventions and achieve better outcomes for patients. ${ }^{28}$ However, despite a growing literature on FL, the majority of studies have been undertaken in Western countries with participants recruited from the general population. ${ }^{21,29,30}$ This knowledge gap can be a barrier to developing management strategies for improving FL of CKD patients with special dietary regimens. Consequently, considering healthcare and dietary differences and the aforementioned gap, it is imperative to explore FL and its influencing factors in Chinese non-dialysis patients with CKD.

\section{Methodology}

\section{Design and Participants}

We conducted a cross-sectional study in a tertiary Hospital from August to December 2020 in China. Research assistants, who were nurses in the Department of Nephrology - were given comprehensive training and instructions about this study and standardized procedures to follow when collecting the questionnaires. The participants gave informed written consent and were invited to complete the self-administered questionnaires while attending the renal clinic. The completion time of our questionnaire was about 15-20 min. Participants were patients with CKD attending follow-up appointments in a renal nurse-led clinic with a multidisciplinary care team including nephrologists, dieticians and specialized nurses. ${ }^{31}$ Regarding the nutritional management in the nurse-led clinic, patients received a comprehensive assessment of nutritional status and a personalized dietary plan based on their disease conditions from the multidisciplinary team at the first time they were seen in the clinic. During the follow-up period, patients could routinely pose nutritional questions and acquire advice on diets from specialty nurses or nephrology physicians. However, when nutritional status deteriorated, CKD patients were transferred to the dietician clinic by physicians to receive more comprehensive nutrition guidance and 
management. In the meantime, if patients had other nutrition-related issues, they could make appointments to see the dieticians by themselves.

Inclusion criteria were as follows: 1) age $\geq 18$ years; 2) diagnosed with CKD by a nephrology specialist; 3) under regular follow-up in this renal clinic, and 4) able to speak Mandarin. The following patients were excluded: 1) diagnosed with life-threatening conditions (eg, acute respiratory distress syndrome or acute heart failure); 2) receiving dialysis treatment or having undergone a kidney transplantation surgery, and 3) unable to complete the survey due to cognitive impairment or other disabilities. Based on the Kidney Disease: Improving global outcome (KDIGO) clinical practice guidelines, ${ }^{32}$ patients were classified by estimated glomerular filtration rate (eGFR), as CKD stage 1 (eGFR $\geq 90 \mathrm{~mL} /$ $\mathrm{min} / 1.73 \mathrm{~m}^{2}$ ), stage $2(\mathrm{eGFR}=60-89)$, stage 3 (eGFR=30-59), stage 4 (eGFR 15-29), or stage 5 (eGFR $\left.<15\right)$.

\section{Sample Size}

Because there are limited empirical studies about FL in individuals with chronic diseases, the sample size was estimated as 200 participants, based on the rule of thumb suggested by Harrell, ${ }^{33}$ at least 10 subjects per variable in the linear regression model. Considering a $10 \%$ rate of invalid questionnaires, the sample size was estimated as 220 .

\section{Outcome Measurements}

Demographic (age, gender, income and marital status) and clinical data (comorbidities, CKD stage and diagnosis period) were collected via self-report questionnaires or electronic medical records from the hospital. Satisfaction with dietary advice from healthcare professionals was assessed by one question with a 5-point Likert scale. ${ }^{34} \mathrm{~A}$ higher score represents greater satisfaction. The points associated with each scale were as follows: $1=$ "very dissatisfied" to $5=$ "very satisfied." Compliance with dietary advice from healthcare professionals was also measured by a self-report question using a 5-point Likert scale ${ }^{34}$ where $1=$ "not at all compliant" to $5=$ "extremely compliant. The recall period of these assessments of satisfaction and compliance with dietary advice was within 1 year.

Food literacy was assessed using a modified Short Food Literacy Questionnaire for Adults developed by Corinna et al. ${ }^{35}$ The questionnaire represents the key functional, interactive, and critical elements of FL. It has been used to assess the FL in individuals with different educational level or has been modified to be used in patients with chronic disease. ${ }^{36,37}$ It is a 12-item questionnaire yields a total score ranging from 7 to 52 . Participants were asked to choose from 4- or 5-point Likert scales that ranged from "terrible" to "very good," "strongly disagree" to "strongly agree," "very difficult" to "very easy," "very tough" to "very easy," or "never" to "always." A higher score represents a higher FL level. The Cronbach's coefficient was 0.82 . Three items-items 3,4, and 5-were modified slightly to be appropriate for Chinese patients with CKD, and they were finally revised into "How familiar are you with the Chinese Food Pyramid?" "I know the official Chinese recommendations about fruit and vegetable consumption," and "I know the official Chinese recommendations about salt intake." In this study, the Cronbach's $\alpha$ of the modified questionnaire was 0.83 .

Motivation for controlling diet was assessed using the Motivation for Dietary Self-Control scale, developed by Hamish et al. ${ }^{38}$ The scale has 11 items with a total score of 78. Each item was rated on a seven-point questionnaire anchored at $0=$ does not describe me at all to $6=$ completely describes me. Items 3.7 and 11 were scored in reverse, with higher scores representing higher levels of dietary restraint. The Cronbach's $\alpha$ of the scale in Chinese population was $0.83 .^{39}$ In this study, the Cronbach's coefficient was 0.73 .

Satisfaction with dietary behaviors was evaluated using the Satisfaction with Dietary Behavior Scale developed by Hamish et al. ${ }^{29}$ The scale comprises eight items with a total range of 0 to 48 . The second item was scored in reverse. A higher total score indicates a greater degree of satisfaction with dietary behavior. The scores represent ratings on a 7-point Likert scale using anchors of "does not describe me at all" (0) to "completely describes me" (6). The Cronbach's $\alpha$ of this scale in Chinese population was $0.91 .{ }^{39}$ In this study, the Cronbach's $\alpha$ was 0.86 .

HISB was measured by four items developed by Maryam et al. ${ }^{40}$ The total score for the four items was 20 . Each item was rated on a 5 -point scale with $5=$ always to $1=$ never. A higher score indicates more active information-seeking behavior. The Cronbach's $\alpha$ was 0.79 for Chinese patients. ${ }^{27}$ In this study, Cronbach's $\alpha$ was 0.75 .

The Short Food Literacy Questionnaire for Adults was translated into Chinese using forward and back-translation. ${ }^{41}$ Two independent translators (one nephrology nurse and one nephrology physician) familiar with the field's terminology completed 
the forward translation task. Next, the scales were translated back to English by an independent translator (one international $\mathrm{Ph} . \mathrm{D}$. candidate) whose native language was English and who had no knowledge of the questionnaire. An expert panel consisting of renal dieticians, nephrology physicians, and specialized nurses reviewed the text and reached consensus regarding the process. Six external experts (two nephrology professors and four dieticians) were invited to review and assess the content validity index (CVI) of these questionnaires. The SFLQ of Item-CVI (I-CVI) was $0.80 \sim 1.00$ and its Scale-CVI /Average (S-CVI/Ave) was 0.96 . The results of I-CVI and S-CVI/Ave exceeded the recommended levels of 0.78 and $0.80 .{ }^{42}$ Pre-testing and cognitive debriefing interviews were accomplished with inpatients before the final revised version was completed. To assess the reliability of the questionnaire, 40 patients with CKD were recruited to complete the questionnaire in a pilot study. The results showed that the Cronbach's $\alpha$ coefficient for the three scales (food literacy, motivation for dietary self-control, and satisfaction with dietary behavior) were 0.78 .

\section{Ethical Issues}

All procedures performed in this study involving human participants followed the ethical standards of the institutional and national research committee and the 1964 Declaration of Helsinki and its later amendments or comparable ethical standards. All participants gave their informed written consent, and the study was approved by the research ethics committee of the hospital [NO.2019(413)].

\section{Data Analysis}

Descriptive statistics such as means and standard deviations were calculated for continuous variables, and frequency and percentage were calculated for categorical data. The univariate analysis was conducted using t-tests or ANOVA tests. Spearman correlation analysis was used for bivariate analysis. The variables with $p<0.1$ in the univariate analysis were added into the multivariable regression model. A stepwise selection approach was adopted to determine the final multivariable linear regression model. IBM SPSS Version 25.0 was used for statistical analysis, and $p<0.05$ was considered statistically significant.

\section{Results}

We interviewed 203 patients with CKD from August to December 2020. Their demographic characteristics and disease history are shown in Table 1. The mean age was 44.5 years (range 18 to 75 ), and $42.5 \%$ were male. Approximately half (50.2\%) were in stages $1-2$ of CKD. The mean FL score was $38.75 \pm 0.38$. The results showed that educational level was associated with $\mathrm{FL}$ scores $(\mathrm{F}=3.763, p<0.05)$. However, we found that the variables of gender, age, marital status, employment status, frequency of visiting a dietary clinic (within 1 year), CKD duration, CKD stage, and follow-up duration were not associated with differences in FL scores.

The correlation analysis showed that compliance with dietary advice $(\mathrm{r}=0.181, p=0.010)$, satisfaction with dietary advice $(\mathrm{r}=0.144, p=0.041)$, HISB $(\mathrm{r}=0.185, p=0.008)$, motivation for dietary self-control $(\mathrm{r}=0.311, p<0.001)$, and satisfaction with dietary behaviors $(\mathrm{r}=0.442, p<0.001)$ were significantly associated with FL score (Table 2$)$.

We used multivariable linear regression to further explore the relationships between these variables and the FL score. The results show that educational level $(\beta=0.221, p=0.004)$, HISB $(\beta=0.146, p=0.019)$, motivation for dietary selfcontrol $(\beta=0.198, p=0.003)$, and satisfaction with dietary behaviors $(\beta=0.319, p<0.001)$ were independent predictors for an increased FL score. The detailed results are shown in Table 3.

\section{Discussion}

FL stems largely from an evolving understanding of health literacy. In general, FL is seen as a complex set of related skills, knowledge, and behaviors needed to incorporate dietary guidelines in daily life. ${ }^{16}$ The importance of nutritional knowledge for self-management of CKD has been emphasized following its association with other chronic diseases. ${ }^{43}$ Nevertheless, even in developed countries, a substantial proportion of individuals are estimated to have inadequate FL, leading to concerns about their food security associated with poorer dietary adherence and health conditions. ${ }^{44,45}$ Inadequate FL can also lead to problems in understanding how dietary behaviors affect chronic disease development. ${ }^{46}$ Given the relationships between healthy dietary patterns and adherence to dietary recommendations, 
Table I Demographic and Clinical Characteristics of the Sample $(N=203)$

\begin{tabular}{|c|c|c|c|c|c|c|}
\hline & \multicolumn{3}{|c|}{$\mathbf{N} \%$} & \multirow{3}{*}{$\begin{array}{c}\begin{array}{c}\text { FL Score } \\
\text { (Mean } \pm \text { SD) }\end{array} \\
38.08 \pm 5.38 \\
39.16 \pm 5.26\end{array}$} & \multirow{2}{*}{$\begin{array}{c}\mathbf{T} / \mathbf{F} \\
1.155\end{array}$} & \multirow{2}{*}{$\begin{array}{c}\boldsymbol{P} \\
0.248^{\mathrm{a}}\end{array}$} \\
\hline \multirow[t]{2}{*}{ Gender } & Men & 87 & 42.9 & & & \\
\hline & Female & 116 & 57.1 & & & \\
\hline \multirow[t]{4}{*}{ Age } & $18-30$ & 25 & 12.3 & $37.56 \pm 5.97$ & 0.698 & $0.554^{\mathrm{b}}$ \\
\hline & $30-45$ & 81 & 39.9 & $39.25 \pm 5.03$ & & \\
\hline & $45-60$ & 80 & 39.4 & $38.56 \pm 5.34$ & & \\
\hline & $60-75$ & 17 & 8.4 & $38.40 \pm 5.78$ & & \\
\hline \multirow[t]{3}{*}{ Education level } & Primary school or below & 41 & 20.2 & $37.15 \pm 5.57$ & 3.763 & $0.025^{b}$ \\
\hline & High or middle school & 30 & 14.8 & $40.60 \pm 5.00$ & & \\
\hline & Bachelor's degree or above & 132 & 65.0 & $38.75 \pm 5.29$ & & \\
\hline \multirow[t]{3}{*}{ Marital status } & Married & 169 & 83.3 & $38.91 \pm 5.20$ & 1.512 & $0.220^{b}$ \\
\hline & Single & 22 & 10.8 & $37.81 \pm 6.27$ & & \\
\hline & Divorced & 12 & 5.9 & $37.16 \pm 5.52$ & & \\
\hline \multirow[t]{3}{*}{ Individual monthly income (yuan) } & $\leq 2500$ & 51 & 25.1 & $37.82 \pm 5.71$ & 1.087 & $0.339^{\mathrm{b}}$ \\
\hline & $250 \mathrm{I}-5000$ & 65 & 32.0 & $38.70 \pm 5.31$ & & \\
\hline & $>5000$ & 87 & 42.9 & $39.21 \pm 5.09$ & & \\
\hline \multirow[t]{4}{*}{ Employment status } & Full time & 108 & 53.2 & $39.06 \pm 5.03$ & 0.670 & $0.571^{\mathrm{b}}$ \\
\hline & Part time & 33 & 16.3 & $38.04 \pm 5.92$ & & \\
\hline & Unemployed & 29 & 14.3 & $37.79 \pm 5.15$ & & \\
\hline & Retired & 33 & 16.3 & $39.03 \pm 5.84$ & & \\
\hline \multirow[t]{4}{*}{ CKD duration } & $<1$ year & 13 & 6.4 & $39.69 \pm 4.40$ & 0.220 & $0.883^{b}$ \\
\hline & I-5 year & 63 & 31 & $38.60 \pm 5.80$ & & \\
\hline & $>5-10$ year & 67 & 33 & $38.84 \pm 5.31$ & & \\
\hline & $>10$ year & 60 & 29.6 & $38.43 \pm 5.08$ & & \\
\hline \multirow[t]{5}{*}{ CKD stage } & 1 & 48 & 23.7 & $38.7 I \pm 5.04$ & 1.702 & $0.136^{b}$ \\
\hline & 2 & 54 & 26.6 & $39.87 \pm 4.78$ & & \\
\hline & 3 & 64 & 31.5 & $38.06 \pm 5.30$ & & \\
\hline & 4 & 13 & 6.4 & $39.54 \pm 6.63$ & & \\
\hline & 5 & 24 & 11.8 & $37.29 \pm 8.15$ & & \\
\hline \multirow[t]{4}{*}{ Frequency of dietary clinic visits (within I year) } & 0 & 82 & 40.4 & $38.46 \pm 5.24$ & 0.331 & $0.803^{b}$ \\
\hline & $1-5$ & 82 & 40.4 & $39.00 \pm 5.30$ & & \\
\hline & $6-10$ & 26 & 12.8 & $38.12 \pm 5.57$ & & \\
\hline & $>10$ & 13 & 6.4 & $39.46 \pm 5.95$ & & \\
\hline \multirow[t]{3}{*}{ Follow up Duration in nursing clinic } & $<$ I year & 34 & 16.7 & $38.35 \pm 5.15$ & 0.955 & $0.387^{\mathrm{b}}$ \\
\hline & $>1-5$ year & 80 & 39.4 & $38.20 \pm 5.82$ & & \\
\hline & $>5$ year & 89 & 43.8 & $39.28 \pm 4.91$ & & \\
\hline
\end{tabular}

Notes: ${ }^{a}$-test; 'one-way ANOVA.

Abbreviations: CKD, chronic kidney disease;. FL, food literacy.

and the progression of $\mathrm{CKD},{ }^{47}$ it is necessary to learn more about $\mathrm{FL}$ in the $\mathrm{CKD}$ population for appropriate nutrition and disease management.

To the best of our knowledge, this is the first study to investigate food-related health literacy among patients with CKD. The mean of FL score of the study population was 38.75 (range 7, 52), suggesting that there is room for improvement in FL of patients. Similarly, it is comparable with Jun-Hao et al report that $46.3 \%$ of their 218 patients with ESKD had limited nutritional literacy. As noted in previous studies, ${ }^{48}$ the issue of CKD patients with limited FL needs to be addressed. As reported in previous studies, ${ }^{28,49}$ the FL of individuals can be effectively enhanced through education programs or interventions, which favorably contributes to changes in unhealthy dietary behaviors. However, because of time constraints, nutrition education might not always be a priority in clinical practice. ${ }^{50}$ Therefore, nephrology physicians and nurses are advised to assess the FL of CKD patients and ensure the effective delivery of nutritional education for patients in multiple ways, such as providing scientific hybrid online-offline resource or videos, ${ }^{51,52}$ or organizing nutrition-related campaigns. ${ }^{53}$ 
Table 2 The Influential Factors Associated with Food Literacy (FL) Scores Using Bivariate Analysis

\begin{tabular}{|l|c|c|c|}
\hline Variable & Median $\left(\mathbf{P}_{\mathbf{2 5}}, \mathbf{P}_{\mathbf{7 5}}\right)$ & $\boldsymbol{r}_{\mathbf{s}}$ & $\mathbf{p}$ \\
\hline Complied with dietary advice & $4(4,4)$ & 0.181 & 0.010 \\
Satisfied with dietary advice & $5(4,5)$ & 0.144 & 0.041 \\
Health information searching & $13(11,15)$ & 0.185 & 0.008 \\
Motivation for dietary self-control & $53(47,59)$ & 0.311 & $<0.001$ \\
Satisfaction with dietary behavior & $34(29,41)$ & 0.442 & $<0.001$ \\
\hline
\end{tabular}

Notes: $r_{s}=$ Spearman rho coefficients. $P_{25}=25^{\text {th }}$ percentile; $P_{75}=75^{\text {th }}$ percentile.

Table 3 Influential Factors Associated with Food Literacy (FL) Scores Using Multivariate Linear Regression

\begin{tabular}{|l|l|l|l|l|l|l|l|}
\hline \multirow{2}{*}{} & \multicolumn{3}{|l}{} & \multicolumn{2}{c|}{$\begin{array}{c}95 \% \\
\text { Confidence Interval }\end{array}$} \\
\cline { 2 - 9 } & B & Std. Error & Beta & T & Sig. & Lower Bound & Upper Bound \\
\hline Educational level & \multicolumn{2}{|l|}{} & & & & & \\
Primary school or below & Reference & & & & & \\
\hline High or middle school & 3.307 & 1.123 & 0.221 & 2.944 & 0.004 & 1.092 & 5.522 \\
College school or above & 1.509 & 0.831 & 0.135 & 1.814 & 0.071 & -0.131 & 3.148 \\
Satisfaction with dietary behaviors & 0.206 & 0.043 & 0.319 & 4.774 & $<0.001$ & 0.121 & 0.291 \\
Motivation for dietary self-control & 0.119 & 0.040 & 0.198 & 2.961 & 0.003 & 0.040 & 0.198 \\
Health information-seeking behavior & 0.294 & 0.124 & 0.146 & 2.364 & 0.019 & 0.049 & 0.538 \\
\hline
\end{tabular}

Notes: $R^{2}=25.7 \%$; adjusted $R^{2}=23.9 \% ; F=5.587 ; p=0.019$.

The multivariable linear regression analysis results show that educational level is significantly associated with FL. This finding concurs with the study of Rocco et al. ${ }^{54}$ Those with higher education levels were more likely to have stronger FL than people with more limited education. Because of the inherent association between FL and health literacy, it is not surprising that educational attainment was the most important determinant of health literacy that had an impact on FL. ${ }^{55}$ With regard to information-seeking behavior, individuals need to actively searching for food information, particularly nutrition-related information, which is crucial for developing awareness and understanding of nourishing food and to increase procedural knowledge and improve food-related behaviors. ${ }^{56,57}$ Hence, it is easy to see that information-seeking behaviors had a significant influence on the FL of patients with CKD. Notably, in despite of the ease of gaining health information by many ways at present (eg, website, smartphone, and TV), it may be not simple for patients to assess the reliability of this health information. According to recent studies, media has had an impact on awareness and knowledge of nutrition issues of individuals, ${ }^{53}$ but some media information on dietary suggestions was based on low-quality scientific evidence, or even contradictory and misleading content, ${ }^{58}$ which may unfavorably influence nutrition and FL of individuals. Consequently, nephrology professionals should be concerned that poor quality of information probably has a negative effect on FL of patients, leading to their low dietary adherence or other adverse events.

Motivation for controlling diet and satisfaction with dietary behaviors may be important psychological factors in dietary behavior. ${ }^{38}$ Eating-related motivation is critical for applying declarative and procedural knowledge because it allows people to act on their food and nutrition knowledge and abilities. ${ }^{59}$ Although previous research revealed that eating-related motivations does not always predictive of long-term maintenance of healthy dietary changes, ${ }^{60}$ they can moderate the self-control that influences the maintenance. Patients with greater satisfaction with dietary behaviors may be more assertive about acquiring knowledge and skills related to food or cooking, and their FL will probably be improved in the process. As an intricate process, change in health behavior includes many causal factors operated by various mechanisms. While knowledge is a precondition for this change, it is often not enough to change individual behavior, including food-related behavior. ${ }^{61}$ We included two psychological variables - motivation for controlling diet and satisfaction with dietary behaviors - to help identify psychological mechanisms required to achieve FL-related health 
behavior change. These variables were significantly associated with FL in patients with CKD in this study, partially consistent with the work of Thomas et al conceptual framework on FL. ${ }^{22,62}$ Consequently, healthcare workers can educate CKD patients on food-related knowledge and pay attention to patients' psychological status related to dietary behaviors, thereby helping patients adhere to their dietary recommendations.

Our study has both strengths and limitations. To our knowledge, this is the first study to explore FL among Chinese patients with $\mathrm{CKD}$, contributing valuable results for further research. We attempted to verify the association between FL and psychological factors, providing references and guidance for nutrition education of patients with CKD. However, the first limitation of our study was the cross-sectional design, which led to difficulty in explaining the causal relations between the variables. Second, all participants were recruited from one medical center in China and the relatively small sample size limits generalizability for clinical practice in other populations. Third, the use of self-report questionnaires can cause discrepancies when assessing FL because at present there are different FL instruments. Additionally, dietary compliance of participants was assessed by self-reported questions, not any objective markers, which may also lead to certain bias. In future, there is a need to develop an FL scale specifically designed for patients with renal disease, and such a scale could better measure and distinguish their functional, interactive, and critical FL. Last but not least, we expected that longitudinal studies will be warranted to verify the causality of these factors and to demonstrate the link between FL and health outcomes to provide more references for making intervention strategies to improve FL of CKD patients.

\section{Conclusions}

Our study showed that there is room for improvement of FL in non-dialysis CKD patients. Patients had higher education levels exhibit more active information-seeking behaviors, have greater satisfaction with dietary behaviors, and their motivation for dietary self-control are more likely to have better FL. Therefore, healthcare workers should be aware of psychosocial factors and information-seeking behaviors in FL and attempt to integrate the assessment of FL into routine food-related education of CKD patients. The findings provide some beneficial insights for healthcare workers on enhancing FL as they manage the nutrition in this patient population.

\section{Author Contributions}

All authors made substantial contributions to conception and design, acquisition of data, or analysis and interpretation of data; took part in drafting the article or revising it critically for important intellectual content; agreed to submit to the current journal; gave final approval of the version to be published; and agree to be accountable for all aspects of the work.

\section{Disclosure}

The authors declare they have no competing interests in this work.

\section{References}

1. de Boer IH, Caramori ML, Chan Juliana CN, et al. Executive summary of the 2020 KDIGO Diabetes Management in CKD guideline: evidence-based advances in monitoring and treatment. Kidney Int. 2020;98(4):839-848. doi:10.1016/j.kint.2020.06.024

2. Wang H, Naghavi M, Allen C, et al. Global, regional, and national life expectancy, all-cause mortality, and cause-specific mortality for 249 causes of death, 1980-2015: a systematic analysis for the Global Burden of Disease Study 2015. Lancet. 2016;388:1459-1544. doi:10.1016/S0140-6736(16) 31012-1

3. Collaboration GCKD. Global, regional, and national burden of chronic kidney disease, 1990-2017: a systematic analysis for the Global Burden of Disease Study 2017. Lancet. 2020;395:709-733. doi:10.1016/s0140-6736(20)30045-3

4. Liyanage $\mathrm{T}$, Ninomiya $\mathrm{T}$, Jha $\mathrm{V}$, et al. Worldwide access to treatment for end-stage kidney disease: a systematic review. Lancet. 2015;385:1975-1982. doi:10.1016/S0140-6736(14)61601-9

5. Siener R. Dietary treatment of metabolic acidosis in chronic kidney disease. Nutrients. 2018;10. doi:10.3390/nu10040512.

6. Kalantar-Zadeh K, Fouque D. Nutritional management of chronic kidney disease. N Engl J Med. 2017;377:1765-1776. doi:10.1056/ NEJMra1700312

7. Kalantar-Zadeh K, Tortorici AR, Chen JL, et al. Dietary restrictions in dialysis patients: is there anything left to eat? Semin Dial. 2015;28:159-168. doi:10.1111/sdi.12348

8. Ikizler TA, Burrowes JD, Byham-Gray LD, et al. KDOQI clinical practice guideline for nutrition in CKD: 2020 update. Am J Kidney Dis. 2020;76 (3Suppl 1):S1-S107. doi:10.1053/j.ajkd.2020.05.006 
9. Anderson CAM, Nguyen HA. Nutrition education in the care of patients with chronic kidney disease and end-stage renal disease. Semin Dial. 2018;31:115-121. doi:10.1111/sdi.12681

10. Pereira RA, Alvarenga MS, Avesani CM, Cuppari L. Strategies designed to increase the motivation for and adherence to dietary recommendations in patients with chronic kidney disease. Nephrol Dial Transplant. 2020;2173-2181. doi:10.1093/ndt/gfaa177

11. Palmer SC, Maggo JK, Campbell KL, et al. Dietary interventions for adults with chronic kidney disease. Cochrane Database Syst Rev. 2017;4: Cd011998. doi:10.1002/14651858.CD011998.pub2

12. Taggart J, Williams A, Dennis S, et al. A systematic review of interventions in primary care to improve health literacy for chronic disease behavioral risk factors. BMC Fam Pract. 2012;13:49. doi:10.1186/1471-2296-13-49

13. Brown M. How to increase patient adherence to therapy. Cutis. 2016;98:E24-E25.

14. Warsame F, Haugen CE, Ying H, et al. Limited health literacy and adverse outcomes among kidney transplant candidates. Am J Transplant. 2019;19(2):457-465. doi:10.1111/ajt.14994

15. Schrauben SJ, Hsu JY, Wright Nunes J, et al. Health behaviors in younger and older adults with CKD: results from the CRIC study. Kidney Int Rep. 2019;4:80-93. doi:10.1016/j.ekir.2018.09.003

16. Vidgen HA, Gallegos D. Defining food literacy and its components. Appetite. Ma. 2014;76:50-59. doi:10.1016/j.appet.2014.01.010

17. West EG, Lindberg R, Ball K, McNaughton SA. The role of a food literacy intervention in promoting food security and food literacy-OzHarvest's NEST Program. Nutrients. 2020;12:2197. doi:10.3390/nu12082197

18. Razavi AC, Dyer A, Jones M, et al. Achieving dietary sodium recommendations and atherosclerotic cardiovascular disease prevention through culinary medicine education. Nutrients. 2020;12:3632. doi:10.3390/nu12123632

19. Boslooper-Meulenbelt K, Patijn O, Battjes-Fries MCE, Haisma H, Pot GK, Navis GJ. Barriers and facilitators of fruit and vegetable consumption in renal transplant recipients, family members and healthcare professionals-a focus group study. Nutrients. 2019;11:2427. doi:10.3390/nu11102427

20. Crews DC, Kuczmarski MF, Miller ER 3rd, Zonderman AB, Evans MK, Powe NR. Dietary habits, poverty, and chronic kidney disease in an urban population. J Ren Nutr. 2015;25:103-110. doi:10.1053/j.jrn.2014.07.008

21. Begley A, Paynter E, Butcher LM, Dhaliwal SS. Examining the association between food literacy and food insecurity. Nutrients. 2019;11:207. doi:10.3390/nu11020445

22. Rosas R, Pimenta F, Leal I, Schwarzer R. FOODLIT-PRO: food literacy domains, influential factors and determinants-a qualitative study. Nutrients. 2019;12:88. doi:10.3390/nu12010088

23. Cullen T, Hatch J, Martin W, Higgins JW, Sheppard R. Food literacy: definition and framework for action. Can J Diet Pract Res. 2015 ;76:140-145. doi:10.3148/cjdpr-2015-010

24. Thomas HM, Irwin JD. Cook It Up! A community-based cooking program for at-risk youth: overview of a food literacy intervention. BMC Res Notes. 2011;4:495. doi:10.1186/1756-0500-4-495

25. Poelman MP, Dijkstra SC, Sponselee H, et al. Towards the measurement of food literacy with respect to healthy eating: the development and validation of the self perceived food literacy scale among an adult sample in the Netherlands. Int J Behav Nutr Phys Act. 2018;15:54. doi:10.1186/ s12966-018-0687-z

26. Tang $\mathrm{C}$, Wu X, Chen X, Pan B, Yang X. Examining income-related inequality in health literacy and health-information seeking among urban population in China. BMC Public Health. 2019;19:221. doi:10.1186/s12889-019-6538-2

27. Zhang X, Zhang R, Lu X. Exploring the effects of patient activation in online health communities on patient compliance. Telemed $J E$ Health. 2020;26:1373-1382. doi:10.1089/tmj.2019.0258

28. Savarese M, Sapienza M, Acquati GM, et al. Educational interventions for promoting food literacy and patient engagement in preventing complications of type 2 diabetes: a systematic review. J Pers Med. 2021;11:795. doi:10.3390/jpm11080795

29. Charlebois S, Music J, Faires S. The impact of COVID-19 on Canada's food literacy: results of a cross-national survey. Internat J Environ Res Public Health. 2021;18:5485. doi:10.3390/ijerph18105485

30. Luta X, Hayoz S, Gréa Krause C, et al. The relationship of health/food literacy and salt awareness to daily sodium and potassium intake among a workplace population in Switzerland. Nutr Metab Cardiovasc Dis. 2018;28:270-277. doi:10.1016/j.numecd.2017.10.028

31. Dengyan M, Yingjun Z, Yi C, Yongshu D. Application of multidisciplinary integration model in management of outpatient follow up in patients with chronic kidney disease. Chinese Nurs Res. 2016;30:3017-3020. doi:10.3969/j.issn.1009-6493.2016.24.022

32. Floege J, Barbour SJ, Cattran DC, et al. Conference Participants. Management and treatment of glomerular diseases (part 1): conclusions from a Kidney Disease: improving Global Outcomes (KDIGO) Controversies Conference. Kidney Int. 2019;95(2):268-280. doi:10.1016/j. kint.2018.10.018

33. Harrell FE Jr. Regression Modeling Strategies. Springer-Verlag; 2001.

34. Sayer RD, Peters JC, Pan Z, Wyatt HR, Hill JO. Hunger, food cravings, and diet satisfaction are related to changes in body weight during a 6-month behavioral weight loss intervention: the Beef WISE Study. Nutrients. 2018;10:700. doi:10.3390/nu10060700

35. Gréa Krause C, Beer-Borst S, Sommerhalder K, Hayoz S, Abel T. A short food literacy questionnaire (SFLQ) for adults: findings from a Swiss validation study. Appetite. 2018;120:275-280. doi:10.1016/j.appet.2017.08.039

36. Rafferty AJ, Hall R, Johnston CS. A novel mobile app (Heali) for disease treatment in participants with irritable bowel syndrome: randomized controlled pilot trial. original paper. J Med Internet Res. 2021;23:e24134. doi:10.2196/24134

37. Sponselee HCS, Kroeze W, Poelman MP, Renders CM, Ball K, Steenhuis IHM. Food and health promotion literacy among employees with a low and medium level of education in the Netherlands. BMC Public Health. 2021;21:1273. doi:10.1186/s12889-021-11322-6

38. Love H, Bhullar N, Schutte NS. Psychological aspects of diet: development and validation of three measures assessing dietary goal-desire incongruence, motivation, and satisfaction with dietary behavior. Appetite. 2019;138:223-232. doi:10.1016/j.appet.2019.03.018

39. Li X, Shan Y, Gao Y, et al. The cross-cultural adaptation and the reliability test for the Chinese-version Dietary Behavior and Psychological Series Scales in maintenance hemodialysis patients. Patient Prefer Adherence. 2021;15:1903-1912. doi:10.2147/ppa.S322868

40. Khazaee-Pool M, Majlessi F, Montazeri A, Pashaei T, Gholami A, Ponnet K. Development and psychometric testing of a new instrument to measure factors influencing women's breast cancer prevention behaviors (ASSISTS). BMC Womens Health. 2016;16:40. doi:10.1186/s12905-016$0318-2$

41. World Health Organization. Process of translation and adaptation of instruments; 2020. Available at: http://www.who.int/substance_abuse/ research_tools/translation/en/. Accessed February 10, 2022. 
42. Polit DF, Beck CT. The content validity index: are you sure you know what's being reported? Critique and recommendations. Res Nurs Health. 2006;29:489-497. doi:10.1002/nur.20147

43. Hakami R, Gillis DE, Poureslami I, FitzGerald JM. Patient and professional perspectives on nutrition in chronic respiratory disease self-management: reflections on nutrition and food literacies. Health Lit Res Pract. 2018;2:e166-e174. doi:10.3928/24748307-20180803-01

44. Nyambayo I. Food security in developed countries (Europe and USA)-is it insecurity and insufficiency or hunger and poverty in developed countries? BAOJ Nutrition. 2015;1:1-7.

45. Schiff R, Freill H. Improving access to phosphorus- and sodium-restricted foods for people living with chronic kidney disease in remote First Nations. Rural Remote Health. 2020;20:5485. doi:10.22605/rrh5485

46. Persoskie A, Hennessy E, Nelson WL. US Consumers' understanding of nutrition labels in 2013: the importance of health literacy. Prev Chronic Dis. 2017;14:E86. doi:10.5888/pcd14.170066

47. Lu L, Huang YF, Wang MQ, et al. Dietary fiber intake is associated with chronic kidney disease (CKD) progression and cardiovascular risk, but not protein nutritional status, in adults with CKD. Asia Pac J Clin Nutr. 2017;26:598-605. doi:10.6133/apjcn.072016.08

48. Lim J-H, Chinna K, Khosla P, Karupaiah T, Daud ZAM. Understanding how nutrition literacy links to dietary adherence in patients undergoing maintenance hemodialysis: a theoretical exploration using partial least squares structural equation modeling. Int J Environ Res Public Health. 2020;17:7479.

49. Butcher LM, Platts JR, Le N, McIntosh MM, Celenza CA, Foulkes-Taylor F. Can addressing food literacy across the life cycle improve the health of vulnerable populations? A case study approach. Health Promot J Austr. 2021;32(Suppl 1):5-16. doi:10.1002/hpja.414

50. Stevenson J, Tong A, Campbell KL, Craig JC, Lee VW. Perspectives of healthcare providers on the nutritional management of patients on haemodialysis in Australia: an interview study. BMJ Open. 2018;8:e020023. doi:10.1136/bmjopen-2017-020023

51. Rabassa M, Alonso-Coello P, Casino G. Nutrimedia: a novel web-based resource for the general public that evaluates the veracity of nutrition claims using the GRADE approach. PLoS One. 2020;15:e0232393-e0232393. doi:10.1371/journal.pone.0232393

52. Thornton J. Clinicians are leading service reconfiguration to cope with covid-19. BMJ. 2020;369:m1444. doi:10.1136/bmj.m1444

53. Lee CJ, Nagler RH, Wang N. Source-specific exposure to contradictory nutrition information: documenting prevalence and effects on adverse cognitive and behavioral outcomes. Health Commun. 2018;33:453-461. doi:10.1080/10410236.2016.1278495

54. Palumbo R, Adinolfi P, Annarumma C, et al. Unravelling the food literacy puzzle: evidence from Italy. Food Policy. $2019 ; 83: 104-115$.

55. Stormacq C, Van den Broucke S, Wosinski J. Does health literacy mediate the relationship between socioeconomic status and health disparities? Integrative review. Health Promot Int. 2019;34:e1-e17. doi:10.1093/heapro/day062

56. Rosas R, Pimenta F, Leal I, Schwarzer R. FOODLIT-PRO: conceptual and empirical development of the food literacy wheel. Int J Food Sci Nutr. 2021;72:99-111. doi:10.1080/09637486.2020.1762547

57. Ghweeba M, Lindenmeyer A, Shishi S, Abbas M, Waheed A, Amer S. What predicts online health information-seeking behavior among Egyptian Adults? A cross-sectional study. J Med Internet Res. 2017;19:e216. doi:10.2196/jmir.6855

58. Gholizadeh Z, Papi A, Ashrafi-Rizi H, Shahrzadi L, Hasanzadeh A. Quality evaluation of Persian nutrition and diet therapy websites. J Educ Health Promot. 2017;6:48. doi:10.4103/jehp.jehp_83_14

59. Ronto R, Ball L, Pendergast D, Harris N. Adolescents' perspectives on food literacy and its impact on their dietary behaviours. Appetite. 2016;107:549-557. doi:10.1016/j.appet.2016.09.006

60. Pelletier LG, Dion SC, Slovinec-D’Angelo M, Reid R. Why Do you regulate what you eat? Relationships between forms of regulation, eating behaviors, sustained dietary behavior change, and psychological adjustment. Motivation Emotion. 2004;28:245-277. doi:10.1023/B: MOEM.0000040154.40922.14

61. Vaitkeviciute R, Ball LE, Harris N. The relationship between food literacy and dietary intake in adolescents: a systematic review. Public Health Nutr. 2015;18:649-658. doi:10.1017/s1368980014000962

62. Thomas H, Azevedo Perry E, Slack J, et al. Complexities in conceptualizing and measuring food literacy. J Acad Nutr Diet. 2019;119:563-573. doi:10.1016/j.jand.2018.10.015

\section{Publish your work in this journal}

Patient Preference and Adherence is an international, peer-reviewed, open access journal that focusing on the growing importance of patient preference and adherence throughout the therapeutic continuum. Patient satisfaction, acceptability, quality of life, compliance, persistence and their role in developing new therapeutic modalities and compounds to optimize clinical outcomes for existing disease states are major areas of interest for the journal. This journal has been accepted for indexing on PubMed Central. The manuscript management system is completely online and includes a very quick and fair peer-review system, which is all easy to use. Visit http://www.dovepress.com/testimonials.php to read real quotes from published authors.

Submit your manuscript here: https://www.dovepress.com/patient-preference-and-adherence-journal 